\title{
RELACIONES VIOLENTAS DE PAREJA EN ESTUDIANTES UNIVERSITARIOS Y SU ASOCIACIÓN CON LA FORMACIÓN EN GÉNERO
}

\section{VIOLENT PARTNER RELATIONSHIPS IN UNIVERSITY STUDENTS AND THEIR ASSOCIATION WITH GENDER TRAINING}

Juan Francisco Rubio Laborda1, Pilar Almansa Martínez²,3, Julian Jesús Arense Gonzalo4, María del Mar Pastor Bravo2,3

\footnotetext{
1Universidad de Murcia. Escuela Universitaria de Enfermería de Murcia. España.

2Departamento de Enfermería. Universidad de Murcia. España.

3IMIB-Arrixaca.
}

${ }^{4}$ Departamento de ciencia sociosanitarias. Universidad de Murcia.

Rubio Laborda, J., Almansa Martínez, P., Arense Gonzalo, J., \& Pastor Bravo, M. (2020). RELACIONES VIOLENTAS DE PAREJA EN ESTUDIANTES UNIVERSITARIOS Y SU ASOCIACIÓN CON LA FORMACIÓN EN GÉNERO. Revista Ene De EnfermeríA, 14(2). Consultado de http://www.ene-enfermeria.org/ojs/index.php/ENE/article/view/944 


\section{Resumen}

Las relaciones violentas de pareja dependen de los procesos de aprendizaje de género que se reflejan en la educación formal. Por ello se ha seleccionado una muestra de estudiantes universitarios de Enfermería categorizados en grupos según formación o la no formación recibida en materia de relaciones de género. Para lo cual, se usa un método transversal descriptivo que propone identificar éste tipo de relaciones violentas en dicha muestra a través de las manifestaciones relacionadas con celos, insultos, sobreprotección, coacción sexual y aislamiento. Dando como resultado asociaciones entre las distintas variables y la formación de género recibida.

Palabras clave: Educación en Enfermería, Violencia de Género, Educación, Violencia de Pareja.

\section{Abstract}

Intimate partner violence is dependent upon the processes of gender role learning, wich is reflected in formal education. Based on this, a sample of nursing degree students were selected and grouped according to whether or not they had received formal education in gender relations. A transversal descriptive metodology was used, with the aim of identifying ths type of abusive relationship within the sample group. Manifested behaviours were observed in relation to jealousy, insults, overprotection, sexual coercion and isolation. The results showed a link between these variables and the level of gender relations education received.

Keywords: Education, Nursing, Gender-Based Violence, Education, Intimate Partner Violence. 


\section{INTRODUCCIÓN}

La violencia en las relaciones de pareja sigue situándose entre los principales problemas de las sociedades actuales generado en base a un paradigma patriarcal que tiene como fundamento principal la subordinación femenina sobre la dominación masculina basándose en relaciones de género y asignando espacios determinados para cada uno de ellos.

Alvarez Dardet (1) indica que para conocer la magnitud del problema se debe realizar una aproximación en base a la incidencia a partir de indicadores judiciales y epidemiológicos.

De ésta forma, si observamos el número de denuncias recopiladas de los indicadores judiciales (2), en la última década ha aumentado exponencialmente, llegando en el año 2017 hasta las 166.260 denuncias.

Para los indicadores epidemiológicos se hace necesario el uso de las macroencuestas de violencia contra la mujer, puesto que ofrecen datos que no son recogidos en las estadísticas oficiales. En éste caso, la macroencuesta del año 2015 (3) muestra que el $12,5 \%$ de las mujeres mayores de 16 años residentes en España han sufrido alguna vez violencia de tipo física o sexual, siendo un $2,7 \%$ en ese mismo año. Sin embargo los cuerpos de seguridad del Estado solo han tenido constancia directa en un $26,8 \%$ de los casos.

Respecto a la violencia de género y su prevalencia en el tiempo, tiene un papel fundamental el aspecto educativo tanto individual como colectivo, a través del cual se ha transmitido durante generaciones el marco ideológico androcentrista en la adolescencia (4), donde existen evidencias de actitudes estereotipadas de género sobre todo en chicos tomando como referencia aspectos corporales, sociales, intelectuales y emocionales. Estas actitudes se mantienen en estadios superiores de educación, incluso en los roles profesionales $(5,6)$. Tendiendo a perpetuarse en relaciones futuras (7).

Sin embargo, un alto nivel de educación formal ha sido considerado como factor protector frente a la violencia de género (8), lo que puede explicar el menor número de estudios de detección de violencia de género en poblaciones universitarias. Por el contrario, Ferreira (9) trata de desmontar el mito de que las personas de alto estatus educativo no tienen tendencia a la violencia en la relación de pareja, ya que la violencia de género puede afectar a cualquier mujer en la medida que es sometida por un hombre.

Ante la controversia respecto al factor protector que supone la educación formal en sufrir o perpetrar la violencia 
de género y la falta de estudios que establezcan si la formación específica en género es un factor protector o a un elemento enmascarador de la violencia de pareja, el objetivo del presente estudio es Identificar relaciones de parejas violentas en estudiantes de Enfermería de la Universidad de Murcia según el grado de formación en género. Esta población permite estudiar el fenómeno de la violencia de género en población con educación formal y compararla con aquella formada en género al recibir formación específica en los niveles de tercero de grado de Enfermería, así como de máster.

\section{MATERIAL Y MÉTOdO}

Diseño transversal descriptivo, donde la unidad de análisis es una comunidad de alto nivel de educación formal, dada su formación universitaria. Así como su comparación con un subgrupo sensibilizado en programas educativos en materia de prevención de violencia de género. La población del estudio se compone de estudiantes de Enfermería de la Universidad de Murcia,y la muestra de sexos fue calculada en función de la población total de estudiantes de Enfermería de todos los cursos de un año lectivo, siendo representativamente mayor del sexo femenino. Se optó por un muestreo aleatorio probabilístico, con elección sistemática de elementos muestrales.
Obteniéndose finalmente 498 sujetos de estudio, de los que 413 eran mujeres y 85 eran hombres.

El instrumento utilizado es una adaptación del cuestionario CADRI o Conflict in Adolescent Dating Relationship Inventory, denominándose "Semáforo de la violencia", que ha sido diseñado y validado en población universitaria española (10). El hecho de que se denomine "Semáforo" viene determinado de sus tres opciones de respuesta: Verde, que es indicativo de "no"; amarillo que expresa "alguna vez" y rojo que implica que "si" existe o ha existido violencia de género. Para un análisis más significativo, en este estudio se transformaron las variables "si" y "a veces" en una sola variable con nombre "si".

El test consta de 10 preguntas diferenciadas en función de que se dirijan a ellas o a ellos, y que deben contestar en referencia a cualquier relación de pareja que hayan mantenido hasta el momento y que tiene como objetivo evidenciar la posible violencia de género que pueden estar sufriendo o ejerciendo. Las preguntas tienen como base los principios de la violencia de género: Críticas a su forma de vestir, control, aislamiento, acoso sexual, celos, sobreprotección y ofensas.

Las preguntas son las siguientes en función de si ha de ser respondida 
por ella o por él y por lo tanto, sufrida o ejercida.

Pregunta 1 (¿Critica tu forma de vestir, de arreglarte y trata de convencerte para que cambies tu aspecto? o ¿Crees que le gusta ir provocando porque se pone una ropa que ella ha elegido o se arregla como quiere?)

Pregunta 2 (¿Te impide ir donde quieras y con quién quieras? o ¿Le impides que decida cuándo, dónde y con quién ir porque piensas que si te quiere a ti no debe salir con nadie más?)

Pregunta 3 (¿Intenta que te alejes de tu familia o de tus amistades o las critica y descalifica? o ¿Te molesta que mantenga buenas y sólidas relaciones con su familia y sus amistades?)

Pregunta 4 ( $¿ T e$ hace sentir inferior, tonta o inútil, o se burla de tus creencias? o ¿Te burlas de lo que piensa, de lo que hace y/o de su trabajo?)

Pregunta 5 ( ¿Te insulta o se dirige a ti con nombres ofensivos? o ¿Le insultas $o$ te diriges a ella con nombres ofensivos?)

Pregunta 6 ( $¿$ Te ignora, te castiga o muestra indiferencia con el silencio? o ¿La ignoras, te muestras indiferente o la castigas con el silencio cuando quieres demostrarle tu enfado?)

Pregunta 7 (¿Se pone celoso y te acusa de mantener relaciones con otras personas? o ¿Te pones celoso y la acu- sas de mantener relaciones con otras personas?)

Pregunta 8 (¿Se muestra muy sobreprotector contigo? o ¿Crees que debes protegerla siempre de todo y de todos porque piensas que ella sola no va a saber o no podrá hacerlo?)

Pregunta 9 ( $¿$ Te llama o manda mensajes constantemente al móvil para saber qué haces, dónde estás y con quién? o ¿La llamas o le mandas mensajes constantemente al móvil para saber qué hace, dónde y con quién está?)

Pregunta 10 ( $¿$ Te obliga a mantener relaciones sexuales o muestra insistencia hasta que cedes para que se calle o porque te exige una "prueba de amor" y tienes miedo de perderle? o ¿Si ella no quiere mantener relaciones sexuales sigues insistiéndole porque piensas que el "no" puede ser un "quizás" o le amenazas con romper la relación porque no te "demuestra su amor"?)

Se realizó también un estudio de fiabilidad del instrumento, para lo que se aplicó un Alfa de Cronbach sobre las 10 preguntas de este cuestionario, arrojando un resultado de 0.663 , en el cuestionario aplicado sobre los hombres y 0,7 en el que fue aplicado sobre mujeres. Lo que manifiesta una confiabilidad fuertemoderada del instrumento.

También se recopiló información referente a la edad, sexo, curso acadé- 
mico y año lectivo. Por otra parte el estudio de la variable "cuso académico" nos permite observar las diferencias en materia de formación de género, puesto que dicha formación no se produce en alumnos de primer curso, y si se realiza en alumnos de tercer curso.

La variable "sexo" se ha tomado de manera independiente dada la diferencia en la formulación de los ítems según hombres y mujeres, para caracterizar a la población lo que permite extrapolar las observaciones encontradas a poblaciones similares.

Se realizó una recogida de datos que tuvo lugar en las aulas de la Facultad de Enfermería de la Universidad de Murcia durante el curso lectivo 13/14 hasta el curso 15/16 con selección aleatoria de los cursos muestrales.

Los cuestionarios fueron autoadministrados a los estudiantes que los cumplimentaron en una media de $5 \mathrm{mi}$ nutos en un ambiente tranquilo. Previamente a la cumplimentación de los cuestionarios, se les explicó la finalidad del estudio y la voluntariedad como requisito de participación. Así mismo, se aseguró el carácter anónimo de los informantes bajo codificación numérica.

El análisis de datos se ha realizado mediante el programa estadístico SPSS versión 22, a través del cual se realizaron diversas tablas de contingen- cia, así como la búsqueda de asociación entre los ítems y curso según los sexos de los encuestados; identificando curso como el aspecto a valorar como formación, dado que en primer curso no se realiza ningún tipo de formación de género, pero si una educación formal universitaria. Mientras que en los grupos de tercero y Máster, además de poseer una educación formal universitaria, se les incluyó en el programa de formación de género.

Con la intención de medir la asociación de los diversos ítems y las variables se utilizaron los estadísticos chi cuadrado de Pearson.

Para un mayor rigor metodológico, la recogida de muestra se realizó en una secuenciación de 3 cursos lectivos. Así mismo el amplio rango de edad de la población, otorgó una mayor variabilidad intergeneracional de la población de estudio, con lo que los datos obtenidos ofrecen una mayor validez y representatividad.

\section{RESULTADOS}

Los resultados evidencian una relación significativa entre determinados ítems y la formación, sobre todo en las mujeres de la muestra. La asociación por curso y por ende la diferenciación entre población con formación específicamente en género y la que no recibió formación, permitió observar una mayor canti- 
dad de respuestas afirmativas de forma generalizada en las alumnas sin formación específica en violencia de género.

La población de estudio (Tabla 1) se ha compuesto de 498 estudiantes de edades comprendidas entre los 17 y 53 años. La media de edad fue de 20,75, mientras que la mediana y la moda coincidieron en los 18 años.

Se realizó un análisis de la asociación ítems-curso en las mujeres de la muestra (tabla 2), en la se obtuvieron datos significativos de asociación en las preguntas 6 , (12\% en aquellos sin formación de género y $3,6 \%$ en mujeres con formación sobre violencia de pareja), 7 (14,6\% y $6,3 \%$ respectivamente), 8 $(43,7 \%$ y $22,3 \%$ en mujeres sin formación y con formación de género correspondientemente.) y que hacen referencia a el castigo por indiferencia, los celos, y la sobreprotección. También se ha destacado el ítem 9 que hace referencia al control coercitivo $(24,3 \%$ en las estudiantes sin formación de género y un $8 \%$ en las que sí poseían dicha formación).

En la pregunta 8, la chi es de 15,793 con una significancia de 0,000, manifestando una muy buena asociación entre dicho ítem y la variable curso.

En el ítem 9, la chi cuadrado es de 13,588, con una significancia de 0,000 .
En el caso de la pregunta 6, se ha obtenido una chi cuadrado de Pearson de 6,502 con un nivel de significancia asintótica de 0,011 lo que muestra una gran asociación entre ambas variables. Por otra parte la pregunta 7 muestra una chi de 5,165 con una significancia de 0,023.

Existe una fuerte asociación significativa inversamente proporcional en éstas preguntas, lo que implica que en el primer curso, aquel en el que no se ha recibido formación de género, es mayor el número de respuestas "si" y "a veces", por lo que nos muestra que la formación de género sí que es un factor protector de sufrir relaciones de pareja violentas.

En los resultados en hombres de los análisis de asociación entre la formación de género ofrecida en los cursos de Tercero y máster con respecto a los cursos de primero donde no se impartía, no se evidencia ningún tipo de asociación significativa en ninguno de los ítems propuestos, aunque existe cierta tendencia de respuestas afirmativas de los alumnos de primero con respecto a los de tercero y máster (Tabla 3), de forma similar a lo reflejado en la muestra de mujeres (Figuras 1 y 2 ). Lo cual se manifiesta en el ítem 6, el cual representa el castigo mediante indiferencia, con un $41,9 \%$ de los estudiantes varones sin formación de 
género y un 27,3 de los que si habían recibido dicha formación.

También se evidencia dicha tendencia en los ítems 7 (con un 18,9\% en los hombres sin formación de género), 8 (con un $24,3 \%$ en los hombres sin formación de género y un $9,1 \%$ de aquellos con formación) y 9 (donde un $14,9 \%$ de los que no habían recibido formación sobre violencia de pareja respondieron de forma afirmativa).

Otro aspecto reseñable en los hombres de esta muestra es el que hace referencia al ítem 5, que manifiesta un indicador de insultos y ofensas. En éste, no se evidencian resultados afirmativos tanto en aquellos con formación de género, como en los que no habían recibido esta formación específica.

\section{Discusión}

La relación entre ítems y formación se muestra en consonancia con el estudio de Caro García (11) en estudiantes de Enfermería, donde ponen en evidencia que tienen las mismas creencias que el resto de jóvenes, pero que ante un proceso de formación de género aparecen cambios en las actitudes del alumnado.

De esta forma, entre la población de estudio, y sobre todo en aquellos que no habían recibido una formación espe- cífica en género se mantenían los mismos arquetipos patriarcales basados en los celos, sobreprotección, control coercitivo y castigo por indiferencia, tal y como sucede en el estudio de Mañas Viejo (10), donde no existía dicha formación sobre igualdad de género y violencia de pareja, a pesar de representar a una muestra igualmente universitaria.

Entre las mujeres del estudio de Mañas Viejo, los ítems con mayor impacto de respuestas afirmativas (amarillo y rojo) fueron los ítems 6 (14,2\%), 7 $(12,4 \%)$ y $8(28,1 \%)$. Generando con ello, un patrón similar en los mismos ítems al de este estudio aunque con el diferencial de la segregación en grupos de formación o no formación en género, y que hacen referencia a el castigo por indiferencia, los celos, y la sobreprotección. A estos ítems hay que añadir otro indicador al que no se hace referencia en el estudio de Mañas Viejo y que en este estudio tiene gran peso entre la muestra de mujeres, el cual hace referencia al control coercitivo y se manifiesta por el ítem 9.

Respecto a la población masculina, el castigo mediante indiferencia (ítem 6) es el más representativo en hombres. Este ítem fue ejercido por el $52,6 \%$ de la muestra de hombres de Mañas Viejo, de forma similar a lo representado en la 
muestra sin formación de género de este estudio.

Además, se denota un notable crecimiento con respecto a la muestra de Mañas Viejo, del carácter sobreprotector en sus parejas y del control coercitivo en la muestra analizada en este estudio.

Otros estudios $(7,12,13)$, muestran una mayor tolerancia en jóvenes estudiantes hacia comportamientos relacionados con los celos, el control coercitivo, la indiferencia como método de castigo, así como la manipulación emocional, el hostigamiento y la persecución como resultado de la falsa idea de amor romántico que induce a pensar en una relación de dominio como concepto esencial en una relación amorosa, lo que se relaciona con la Teoría del sexismo ambivalente (14), aunque otro estudio muestra como disminuye la tolerancia y aumenta el conocimiento según el estado civil de la muestra (15).

Por otra parte los resultados de nuestro estudio coinciden con los de García Díaz (16), en los que existían mayoritariamente acciones de desapego y coerción, lo que corresponde a los ítems 6 y 9 del instrumento utilizado.

Como muestran los datos del ítem 5 en hombres; no parece clara la efectividad de la educación de género sobre la prevención de la violencia verbal en los chicos del estudio, violencia que como tal, posee una naturaleza predictiva de la violencia física (17). Aunque no es esta violencia física la que predomine en los jóvenes adultos, sino las agresiones emocionales y los métodos de control coercitivo indirecto (18), que dada su asiduidad en las relaciones de jóvenes suele ser normalizada. Es así como suele comenzar la violencia de género, enmascarada bajo "tácticas coercitivas sutiles" relacionadas con el cuidado y la sobreprotección que se interiorizan en la víctima como manifestaciones mitificadas de amor del agresor (19).

Otro de los mitos que se hacen presentes en la muestra de estudio con mayor intensidad es la presencia de "celos" como método de control psicológico utilizado por los agresores, y que se manifiesta en este estudio mediante el ítem 7. De esta forma, no son los celos la causa de un posterior acto violento, sino que son los celos en sí el propio acto violento (20).

Cantera (21), muestra que las chicas tienden a considerar las amenazas, humillaciones, insultos, presiones para mantener relaciones, celos y control coercitivo como "malas conductas", rehusándose a utilizar el término violencia por considerarlo excesivamente dañino. Así mismo Rugama González (22) en su investigación observó como muchos estudiantes de enfermería no per- 
cibían las agresiones sexuales como violencia sexual. Sin embargo otro estudio realizado por García Díaz (15) en estudiantes de enfermería y relaciones de pareja violentas expone que el 85 ' $8 \%$ de la muestra había sufrido conductas violentas en la relación de pareja, manifestando un 66 ' $3 \%$ de la muestra haber percibido actos de control coercitivo.

Otro estudio evidencia una alta prevalencia de estas relaciones violentas de pareja entre profesionales, docentes y estudiantes de ciencias de la salud, donde se muestran cifras superiores al $50 \%$ de la muestra (23).

De esta forma se hace necesaria la formación de género, especialmente, teniendo en cuenta que el $92,4 \%$ de los profesionales sanitarios no ha recibido nunca una formación específica sobre violencia de género (24), y aún más cuando éstos no identifican la violencia de género como un rol profesional propio, sino como uno delegado o especializado (25).Sin embargo, este estudio muestra una diferenciación significativa en el efecto que tiene esta formación respecto a mujeres y a hombres, donde en estos últimos no se evidencia un gran impacto, al menos a nivel estadístico respecto a las relaciones violentas de pareja, como también se muestra en el estudio de Caro García (26), donde la formación de género es más eficaz en las chicas para deconstrucción de los mitos sobre el amor, y donde también muestra un notable retroceso en los avances conseguidos ante la supresión de formación de género durante un año.

Entre las limitaciones de este estudio se encuentra la posible influencia de la deseabilidad social, la madurez personal de la muestra encuestada dada la relación intergeneracional entre los cursos, y la influencia de la educación no formal y la cultura externa.

\section{CONCLUSIONES}

Parece existir una fuerte asociación entre sufrir actos violentos de pareja, como los celos, la sobreprotección y el control, con la falta de formación en igualdad y violencia de género, tal y como ocurre en las diferencias observadas entre los estudiantes de primer con los de tercer curso y máster. Así, se puede concluir que la formación específica de género es un factor protector en mujeres, de forma que a mayor formación, menor es la violencia sufrida. Por lo que las mujeres con menor formación específica en género constituyen un grupo vulnerable, sobre las cuales deberían ir indicadas las medidas de prevención y protección.

En referencia al sexo femenino, las mujeres sufrieron un alto nivel de asociación en los ítems relacionados con 
el castigo mediante indiferencia, los celos, la sobreprotección y control. Así mismo, en los hombres se mantienen estos mismos indicadores y se añade en menor medida los pensamientos de provocación por la forma de vestir de sus parejas.

Otra evidencia sobre la que reflexionar es que no parece existir una asociación estadísticamente significativa entre la formación de género recibida y los hombres del estudio, lo que induce a pensar que se deberían utilizar otras formas de concienciación más específicos sobre los varones de esta población.

Por lo tanto, el nivel de formación permite disminuir de forma más clara ciertos patrones de violencia de pareja considerados como más sutiles.

Un razonamiento lógico se relaciona con un mecanismo de compensación y autodefensa del pensamiento patriarcal que se manifiesta por aspectos de violencia de género indirecta (casi siempre violencia psicológica) ante la imposibilidad de realizar la violencia de género directa debido a los constructos culturales actuales que relegan los actos de violencia de género, sobre todo aquellos considerados típicamente de mayor gravedad como son las agresiones físicas.

Así, se puede concluir también que la formación específica en género es una herramienta útil en la prevención de la violencia de género. 


\section{BIBLIOGRAFÍA}

1. Álvarez-Dardet, S. M., Pérez Padilla, J., \& Lorence Lara, B. (2013). La violencia de pareja contra la mujer en España: Cuantificación y caracterización del problema, las víctimas, los agresores y el contexto social y profesional. Psychosocial Intervention, 22(1), 41-53.

2. Delegación del gobierno para la violencia de género del ministerio de sanidad, s. s. e. i. (2018). Portal Estadístico. Disponible en: http://estadisticasviolenciagenero.igualdad.mpr.gob.es/

3. Género., D. d. G. p. I. V. d. (2015). Macroencuesta de violencia contra la mujer 2015. Avance de resultados. Disponible en: http://www.violenciagenero.igualdad.mpr.gob.es/violenciaEnCifras/macroencuesta2015/pdf/AVANCE_MACROENCUESTA_VIOLENCIA_CONTRA_LA_MUJER_2015.pdf

4. Bravo, P. C., \& Moreno, P. V. (2007). La interiorización de los estereotipos de género en jóvenes y adolescentes. Revista de Investigación Educativa, 25(1), 35-38.

5. Barberá, E., Candela, C., \& Ramos, A. (2008). Elección de carrera, desarrollo profesional y estereotipos de género. Revista de Psicología Social, 23(2), 275-285.

6. Lajo, M. R., Baños, R. V., \& Niella, M. F. (2008). Barreras de género y actitudes hacia las redes sociales en alumnado universitario de las facultades de educación. Revista de Investigación Educativa, 26(1), 45-72.

7. Bravo, M. d. M. P., Meseguer, C. B., Llor, A. M. S., \& Pina-Roche, F. (2018). Conocimientos, actitudes y prácticas de adolescentes españoles sobre la violencia de pareja. iQual. Revista de Género e Igualdad(1), 145-158.

8. Avedano-Ferrari, D. I. (2017). Programa educativo para la prevención de violencia de género en Educación Secundaria.

9. Ferreira, G. (1989). Mujer maltratada. Un estudio sobre las mujeres víctimas de violencia doméstica.

10.Viejo, C. M., Mas, A. M., Cerdá, M. E., Sánchez, N. M., \& Corbí, R. G. (2012). Exploración de la violencia en las relaciones de pareja de jóvenes universitarias/os.
11.García, C. C., \& Gimeno, M. C. M. (2017). Creencias del amor romántico y violencia de género. International Journal of Developmental and Educational Psychology, 2(1), 47-56.

12.Álvarez, C. D., \& Márquez, E. M. (2011). Evaluación psicométrica de la percepción de la violencia de género en la adolescencia. International Journal of Developmental and Educational Psychology: INFAD. Revista de Psicología, 1(2), 197-206.

13.Roche, F. P., Llor, A. M. S., Bravo, M. d. M. P., \& Meseguer, C. B. (2016). Identificación de la violencia entre adolescentes como mecanismo de control en el aula y en el noviazgo. NURE investigación: Revista Científica de enfermería, 13(82), 4.

14.Glick, P., \& Fiske, S. T. (1996). The Ambivalent Sexism Inventory: Differentiating hostile and benevolent sexism. Journal of personality and social psychology, 70(3), 491

15.Sánchez, C. A. V., Fernández, C. G., \& Díaz, Á. S. (2016). Violencia de género: conocimientos y actitudes de las enfermeras en atención primaria. Atención Primaria, 48(10), 623-631.

16.Díaz, V. G., Feito, A. F., Díaz, F. J. R., González, M. L. L., Díaz, M. d. P. M., \& Pérez, A. L. (2013). Violencia de género en estudiantes de enfermería durante sus relaciones de noviazgo. Atención Primaria, 45(6), 290-296

17.Swart, L.-A., Seedat, M., Stevens, G., \& Ricardo, I. (2002). Violence in adolescents' romantic relationships: findings from a survey amongst schoolgoing youth in a South African community. Journal of adolescence, 25(4), 385-395.

18.Jezl, D. R., Molidor, C. E., \& Wright, T. L. (1996). Physical, sexual and psychological abuse in high school dating relationships: Prevalence rates and self-esteem issues. Child and Adolescent Social Work Journal, 13(1), 69-87.

19.Andrés, A. G., \& Usaola, C. P. (2009). Dificultades en un caso de violencia de género. Archivos de psiquiatría, 72(1), 50-58.

20.Bosch-Fiol, E., \& Ferrer-Pérez, V. A. (2012). Nuevo mapa de los mitos sobre la violencia de género en el siglo XXI. Psicothema, 24(4), 548-554.

21.Cantera, I., Estébanez, I., \& Vázquez, N. (2009). Violencia contra las mujeres jóvenes: la violencia 
psicológica en las relaciones de noviazgo. Informe final.

22.González, R., Milagros, S., Palacios López, K. d. C., Gutiérre, A., \& Ibeth, J. (2017). Percepción de los estudiantes de quinto año de Enfermería, acerca de la violencia hacia la mujer. POLISAL, UNANManagua. II semestre 2016.

23.Moreno-Cubillos, C. L., Sepúlveda-Gallego, L. E., \& Restrepo-Rendon, L. F. (2013). Prevalencia de violencia y discriminación contra la mujer en la Facultad de Ciencias para la Salud, Universidad de Caldas, Colombia, 2010-2011. Revista Colombiana de Obstetricia y Ginecología, 64(1), 12-20.

24.Alcaraz, C. L., Roche, F. P., Hernández, M. T., Meseguer, C. B., Rodríguez, J. D. P., \& Esparza, A.
O. (2014). Formación y detección de la violencia de género en la profesión sanitaria. Revista de Enfermagem da UFSM, 4(1), 217-226.

25.Rigol Cuadra, A., Galbany Estragués, P., Fuentes Pumarola, C., Martí, B., Dolors, M., RodríguezMartín, D., \& Ballester Ferrando, D. (2015). Percepción de los estudiantes de enfermería sobre violencia de pareja: conocimientos, creencias y rol profesional. Revista Latino-Americana de Enfermagem, vol. 23, num. 3, p. 527-534.

26.García, C. C. (2015). Formación en género y detección precoz de la violencia contra las mujeres a partir de las creencias sobre el amor romántico: un estudio cualitativo en jóvenes universitarios/as de grado en enfermería.

\section{TABLAS Y FIGURAS}

Tabla 1. Características de la muestra

\begin{tabular}{|c|c|c|c|c|}
\hline & & No & $\mathrm{Si}$ & $\mathrm{Chi}^{2}$ de Pearson \\
\hline \multirow[t]{3}{*}{$\mathrm{P} 1$} & Primero & $94,3 \%$ & $5,7 \%$ & 0,774 \\
\hline & \multirow[t]{2}{*}{$3^{\circ}$ y Máster } & \multirow[t]{2}{*}{$92,0 \%$} & \multirow[t]{2}{*}{$8,0 \%$} & Sig. \\
\hline & & & & 0,379 \\
\hline \multirow[t]{3}{*}{$\mathrm{P} 2$} & \multirow[t]{2}{*}{ Primero } & \multirow[t]{2}{*}{$93,7 \%$} & \multirow[t]{2}{*}{$6,3 \%$} & 2,155 \\
\hline & & & & Sig. \\
\hline & $3^{\circ}$ y Máster & $97,3 \%$ & $2,7 \%$ & 0,142 \\
\hline \multirow[t]{3}{*}{ P3 } & \multirow[t]{2}{*}{ Primero } & \multirow[t]{2}{*}{$97,0 \%$} & \multirow[t]{2}{*}{$3,0 \%$} & 0,091 \\
\hline & & & & Sig. \\
\hline & $3^{\circ}$ y Máster & $96,4 \%$ & $3,6 \%$ & 0,764 \\
\hline \multirow[t]{3}{*}{ P4 } & \multirow[t]{2}{*}{ Primero } & \multirow[t]{2}{*}{$96,3 \%$} & \multirow[t]{2}{*}{$3,7 \%$} & 0,237 \\
\hline & & & & Sig. \\
\hline & $3^{\circ}$ y Máster & $97,3 \%$ & $2,7 \%$ & 0,626 \\
\hline \multirow[t]{3}{*}{ P5 } & \multirow[t]{2}{*}{ Primero } & \multirow[t]{2}{*}{$98,3 \%$} & \multirow[t]{2}{*}{$1,7 \%$} & 0,337 \\
\hline & & & & Sig. \\
\hline & $3^{\circ}$ y Máster & $99,1 \%$ & $0,9 \%$ & 0,562 \\
\hline \multirow[t]{3}{*}{ P6 } & \multirow[t]{2}{*}{ Primero } & \multirow[t]{2}{*}{$88,0 \%$} & \multirow[t]{2}{*}{$12,0 \%$} & 6,502 \\
\hline & & & & Sig. \\
\hline & $3^{\circ}$ y Máster & $96,4 \%$ & $3,6 \%$ & 0,011 \\
\hline
\end{tabular}




\begin{tabular}{|c|c|c|c|c|}
\hline \multirow[t]{3}{*}{$\mathrm{P} 7$} & \multirow[t]{2}{*}{ Primero } & \multirow[t]{2}{*}{$85,4 \%$} & \multirow[t]{2}{*}{$14,6 \%$} & 5,165 \\
\hline & & & & Sig. \\
\hline & $3^{\circ}$ y Máster & $93,7 \%$ & $6,3 \%$ & 0,023 \\
\hline \multirow[t]{3}{*}{ P8 } & \multirow[t]{2}{*}{ Primero } & \multirow[t]{2}{*}{$56,3 \%$} & \multirow[t]{2}{*}{$43,7 \%$} & 15,793 \\
\hline & & & & Sig. \\
\hline & $3^{\circ}$ y Máster & $77,7 \%$ & $22,3 \%$ & 0,000 \\
\hline \multirow[t]{3}{*}{ P9 } & \multirow[t]{2}{*}{ Primero } & \multirow[t]{2}{*}{$75,7 \%$} & \multirow[t]{2}{*}{$24,3 \%$} & 13,588 \\
\hline & & & & Sig. \\
\hline & $3^{\circ}$ y Máster & $92,0 \%$ & $8,0 \%$ & 0,000 \\
\hline \multirow[t]{3}{*}{ P10 } & \multirow[t]{2}{*}{ Primero } & \multirow[t]{2}{*}{$98,0 \%$} & \multirow[t]{2}{*}{$2,0 \%$} & 0,020 \\
\hline & & & & Sig. \\
\hline & $3^{\circ}$ y Máster & $98,2 \%$ & $1,8 \%$ & 0,888 \\
\hline
\end{tabular}

Tabla 2. Asociación ítems-curso en mujeres

\begin{tabular}{|c|c|c|c|c|}
\hline & & No & $\mathrm{Si}$ & $\mathrm{Chi}^{2}$ de Pearson \\
\hline \multirow[t]{3}{*}{ P1 } & Primero & $94,3 \%$ & $5,7 \%$ & 0,774 \\
\hline & \multirow[t]{2}{*}{$3^{\circ}$ y Máster } & \multirow[t]{2}{*}{$92,0 \%$} & \multirow[t]{2}{*}{$8,0 \%$} & Sig. \\
\hline & & & & 0,379 \\
\hline \multirow[t]{3}{*}{ P2 } & \multirow[t]{2}{*}{ Primero } & \multirow[t]{2}{*}{$93,7 \%$} & \multirow[t]{2}{*}{$6,3 \%$} & 2,155 \\
\hline & & & & Sig. \\
\hline & $3^{\circ}$ y Máster & $97,3 \%$ & $2,7 \%$ & 0,142 \\
\hline \multirow[t]{3}{*}{ P3 } & \multirow[t]{2}{*}{ Primero } & \multirow[t]{2}{*}{$97,0 \%$} & \multirow[t]{2}{*}{$3,0 \%$} & 0,091 \\
\hline & & & & Sig. \\
\hline & $3^{\circ}$ y Máster & $96,4 \%$ & $3,6 \%$ & 0,764 \\
\hline \multirow[t]{3}{*}{$\mathrm{P} 4$} & \multirow[t]{2}{*}{ Primero } & \multirow[t]{2}{*}{$96,3 \%$} & \multirow[t]{2}{*}{$3,7 \%$} & 0,237 \\
\hline & & & & Sig. \\
\hline & $3^{\circ}$ y Máster & $97,3 \%$ & $2,7 \%$ & 0,626 \\
\hline \multirow[t]{3}{*}{ P5 } & \multirow[t]{2}{*}{ Primero } & \multirow[t]{2}{*}{$98,3 \%$} & \multirow[t]{2}{*}{$1,7 \%$} & 0,337 \\
\hline & & & & Sig. \\
\hline & $3^{\circ}$ y Máster & $99,1 \%$ & $0,9 \%$ & 0,562 \\
\hline \multirow[t]{3}{*}{ P6 } & \multirow[t]{2}{*}{ Primero } & \multirow[t]{2}{*}{$88,0 \%$} & \multirow[t]{2}{*}{$12,0 \%$} & 6,502 \\
\hline & & & & Sig. \\
\hline & $3^{\circ}$ y Máster & $96,4 \%$ & $3,6 \%$ & 0,011 \\
\hline
\end{tabular}




\begin{tabular}{|c|c|c|c|c|}
\hline \multirow[t]{3}{*}{$\mathrm{P} 7$} & \multirow[t]{2}{*}{ Primero } & \multirow[t]{2}{*}{$85,4 \%$} & \multirow[t]{2}{*}{$14,6 \%$} & 5,165 \\
\hline & & & & Sig. \\
\hline & $3^{\circ}$ y Máster & $93,7 \%$ & $6,3 \%$ & 0,023 \\
\hline \multirow[t]{3}{*}{ P8 } & \multirow[t]{2}{*}{ Primero } & \multirow[t]{2}{*}{$56,3 \%$} & \multirow[t]{2}{*}{$43,7 \%$} & 15,793 \\
\hline & & & & Sig. \\
\hline & $3^{\circ}$ y Máster & $77,7 \%$ & $22,3 \%$ & 0,000 \\
\hline \multirow[t]{3}{*}{ P9 } & \multirow[t]{2}{*}{ Primero } & \multirow[t]{2}{*}{$75,7 \%$} & \multirow[t]{2}{*}{$24,3 \%$} & 13,588 \\
\hline & & & & Sig. \\
\hline & $3^{\circ}$ y Máster & $92,0 \%$ & $8,0 \%$ & 0,000 \\
\hline \multirow[t]{3}{*}{ P10 } & \multirow[t]{2}{*}{ Primero } & \multirow[t]{2}{*}{$98,0 \%$} & \multirow[t]{2}{*}{$2,0 \%$} & 0,020 \\
\hline & & & & Sig. \\
\hline & $3^{\circ}$ y Máster & $98,2 \%$ & $1,8 \%$ & 0,888 \\
\hline
\end{tabular}

Tabla 3. Asociación ítems-curso en hombres

\begin{tabular}{|c|c|c|c|c|}
\hline & & No & $\mathrm{Si}$ & Chi $^{2}$ de Pearson \\
\hline \multirow[t]{3}{*}{ P1 } & Primero & $89,2 \%$ & $10,8 \%$ & 1,313 \\
\hline & \multirow[t]{2}{*}{$3^{\circ}$ y Máster } & \multirow[t]{2}{*}{$100,0 \%$} & \multirow[t]{2}{*}{$0,0 \%$} & Sig. \\
\hline & & & & 0,252 \\
\hline \multirow[t]{3}{*}{ P2 } & \multirow[t]{2}{*}{ Primero } & \multirow[t]{2}{*}{$97,3 \%$} & \multirow[t]{2}{*}{$2,7 \%$} & 0,304 \\
\hline & & & & Sig. \\
\hline & $3^{\circ}$ y Máster & $100,0 \%$ & $0,0 \%$ & 0,581 \\
\hline \multirow[t]{3}{*}{ P3 } & \multirow[t]{2}{*}{ Primero } & \multirow[t]{2}{*}{$98,6 \%$} & \multirow[t]{2}{*}{$1,4 \%$} & 0,150 \\
\hline & & & & Sig. \\
\hline & $3^{\circ}$ y Máster & $100,0 \%$ & $0,0 \%$ & 0,698 \\
\hline \multirow[t]{3}{*}{ P4 } & \multirow[t]{2}{*}{ Primero } & \multirow[t]{2}{*}{$91,9 \%$} & \multirow[t]{2}{*}{$8,1 \%$} & 0,960 \\
\hline & & & & Sig. \\
\hline & $3^{\circ}$ y Máster & $100,0 \%$ & $0,0 \%$ & 0,327 \\
\hline \multirow[t]{3}{*}{ P5 } & \multirow[t]{2}{*}{ Primero } & \multirow[t]{2}{*}{$100,0 \%$} & \multirow[t]{2}{*}{$0,0 \%$} & nv \\
\hline & & & & Sig. \\
\hline & $3^{\circ}$ y Máster & $100,0 \%$ & $0,0 \%$ & nv \\
\hline \multirow[t]{3}{*}{ P6 } & \multirow[t]{2}{*}{ Primero } & \multirow[t]{2}{*}{$58,1 \%$} & \multirow[t]{2}{*}{$41,9 \%$} & 0,853 \\
\hline & & & & Sig. \\
\hline & $3^{\circ}$ y Máster & $72,7 \%$ & $27,3 \%$ & 0,356 \\
\hline
\end{tabular}


Rubio-Laborda, Almansa-Martínez, Arense-Gonzalo, Pastor-Bravo RELACIONES VIOLENTAS DE PAREJA EN ESTUDIANTES UNIVERSITARIOS Y SU ASOCIACIÓN CON LA FORMACIÓN EN GÉNERO

\begin{tabular}{|c|c|c|c|c|}
\hline \multirow[t]{3}{*}{ P7 } & \multirow[t]{2}{*}{ Primero } & \multirow[t]{2}{*}{$81,1 \%$} & \multirow[t]{2}{*}{$18,9 \%$} & 2,491 \\
\hline & & & & Sig. \\
\hline & $3^{\circ}$ y Máster & $100,0 \%$ & $0,0 \%$ & 0,114 \\
\hline \multirow[t]{3}{*}{ P8 } & \multirow[t]{2}{*}{ Primero } & \multirow[t]{2}{*}{$75,7 \%$} & \multirow[t]{2}{*}{$24,3 \%$} & 1,280 \\
\hline & & & & Sig. \\
\hline & $3^{\circ}$ y Máster & $90,9 \%$ & $9,1 \%$ & 0,258 \\
\hline \multirow[t]{3}{*}{ P9 } & \multirow[t]{2}{*}{ Primero } & \multirow[t]{2}{*}{$85,1 \%$} & \multirow[t]{2}{*}{$14,9 \%$} & 1,878 \\
\hline & & & & Sig. \\
\hline & $3^{\circ}$ y Máster & $100,0 \%$ & $0,0 \%$ & 0,171 \\
\hline \multirow[t]{3}{*}{ P10 } & \multirow[t]{2}{*}{ Primero } & \multirow[t]{2}{*}{$93,2 \%$} & \multirow[t]{2}{*}{$6,8 \%$} & 0,790 \\
\hline & & & & Sig. \\
\hline & $3^{\circ}$ y Máster & $100,0 \%$ & $0,0 \%$ & 0,374 \\
\hline
\end{tabular}

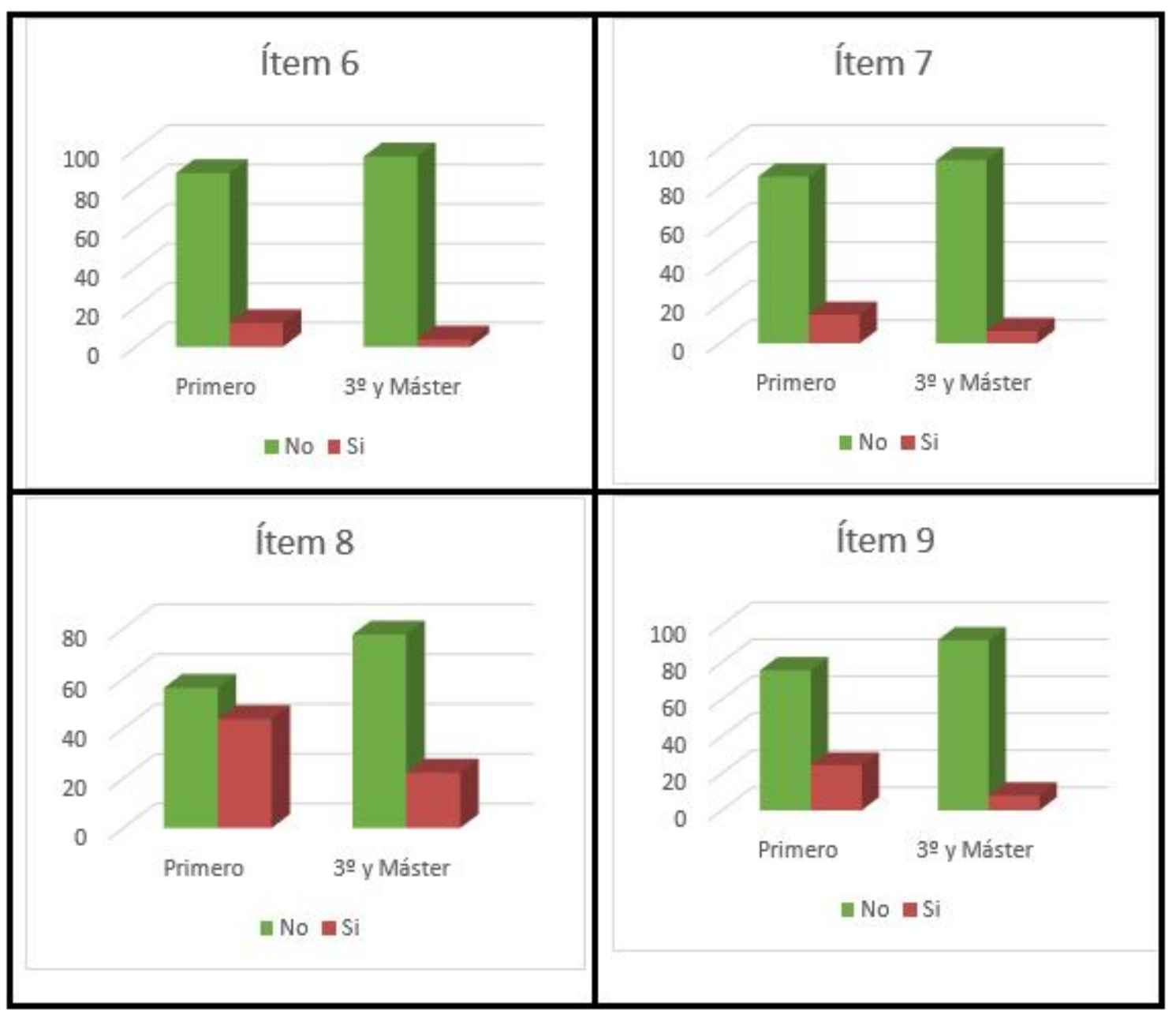

Figura 1. Respuestas de ítems por curso en mujeres 


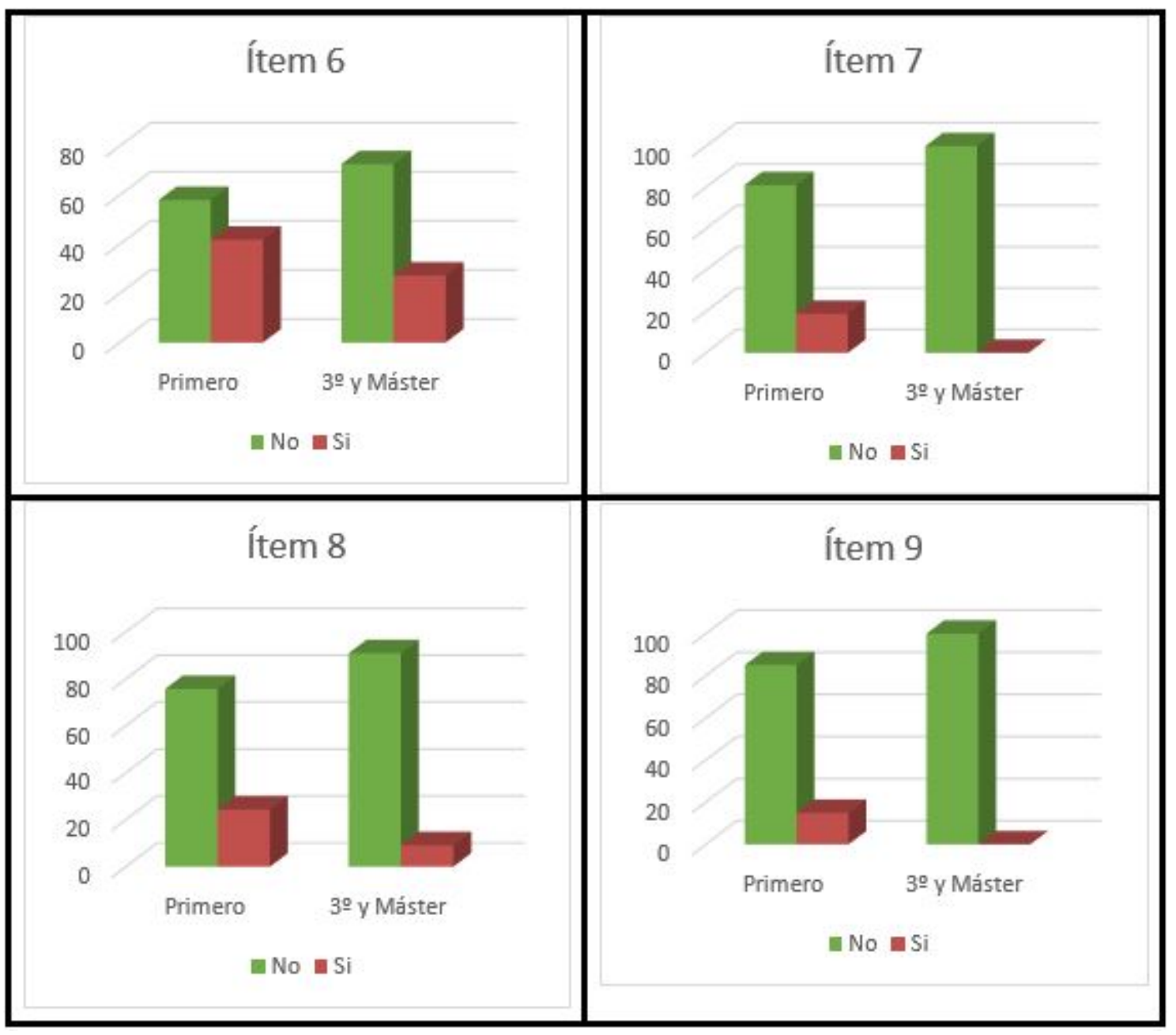

Figura 2. Respuestas de ítems por curso en hombres 